\title{
ARTICLE
}

\section{High temperature liquid metal corrosion and its thermal hydraulic effects in spent fuel transmutation systems}

\author{
Fenglei Niu ${ }^{\mathrm{a}^{*}}$, Li Tian ${ }^{\mathrm{a}}$, Junnan Zhang ${ }^{\mathrm{a}}$, Sheng Gao ${ }^{\mathrm{b}}$ and Yican $\mathrm{Wu}^{\mathrm{b}}$ \\ ${ }^{a}$ State Key Laboratory of Alternate Electrical Power System with Renewable Energy Sources, North China Electric Power University, \\ Beijing 102206, China; ${ }^{b}$ Institute of Nuclear Energy Safety Technology, Chinese Academy of Sciences, Hefei, Anhui, 230031, China
}

\begin{abstract}
The corrosion of Liquid lead or lead-bismuth eutectic (LBE) on the structural steels is a serious problem for their application in fast reactors or accelerator-driven transmutation systems. This paper focuses on the modeling and experimental study on LBE corrosion in the spent fuel transmutation systems, as well as the effects of corrosion products on heat transfer and flow. By studying the corrosion and deposit of the oxidation products on the pipe wall, the empirical correlations of thermal resistance and fouling factor can be theoretically developed and experimentally verified. The comparison of fouling factors obtained with each oxygen level is presented, the relationship between fouling factors and oxygen concentrations is correlated, and the effects of oxidation on heat transfer are demonstrated qualitatively by wetting conditions of the samples.
\end{abstract}

Keywords: LBE; corrosion; oxygen control; fouling factor; spallation neutron target

\section{Introduction}

Liquid lead and Lead bismuth eutectic (LBE) have been primary candidate materials for high-power spallation neutron targets, coolant in accelerator driven systems (ADS) and in advanced nuclear reactors due to its favorable thermal physical and chemical properties, including the low melting point, low vapor pressure, wide margin to boiling, high spallation neutron yield, low neutron moderation and capture, and chemical inertness resulting in mild reaction with air and water. However, the corrosion of LBE on the structural materials has been an obstacle to the application in fast reactors and accelerator-driven transmutation systems. The structural materials are severely corroded if they are exposed to LBE directly at medium to high temperatures, which may lead to material failures and other troubles, even safety problems. Large amount of researches have been done on the modeling and experimental study of LBE corrosion.

To reduce the corrosion, a protective oxide film needs to be built up at the interface of liquid metals and their steel carriers [2]. The traditional method is oxygen injection, which helps to form the protective oxide film. Once the oxide film is formed on the structure surface, the direct dissolution of the structural materials becomes negligible because the diffusion rates of the alloying components are very small in the protective oxide layer [3]. But the gas-phase oxygen control technique has many shortcomings, such as the poor efficiency, the rapid control difficulties, the excess oxide residues, and the sealing problem of the closed LBE loop.

*Corresponding author. Email: niufenglei@ncepu.edu.cn
As for the shortcomings above, solid-phase oxygen control is developed in the recent years. The oxygen mass exchanger is adopted in the solid-phase oxygen control processes in order to achieve rapid adjustment of the oxygen concentration in the liquid metal and avoid the generation of oxide residues at the same time.

The experience of the experiments shows that corrosion and deposit of oxidation product on the pipe wall can still not be avoided. These may affect the processes of heat transfer, so the concept of fouling factor is introduced in the research processes. The comparison of fouling factors obtained with each oxygen level is presented, the relationship between fouling factor and oxygen concentration is correlated, and the effects of oxidation on heat transfer are demonstrated by wetting condition of the samples. The oxygen concentration affects the fouling factors very much. So the application of liquid metal or LBE as a coolant in nuclear system requires a comprehensive consideration of the effects of oxygen on corrosion and Thermal Hydraulic effects.

\section{Mechanism and mitigation of corrosion processes}

Dissolution of steel components into the liquid metal is the primary modus of corrosion of steels exposed to LBE when there is no erosion due to the flow. The main driving force for liquid metal corrosion is the chemical potential for dissolution of all solid surfaces in contact with the liquids [4]. So the solubility of the components of steel such as the iron, the chromium and the nickel in LBE plays an important role in the corrosion processes, 
the experimental result shows that the solubility of $\mathrm{Ni}$ in LBE is much higher than that of Fe and Cr. The different solubility of the materials leads to selective dissolution and intergranular corrosion, which may cause the failure of structural materials eventually. The dissolution rate depends on the liquid media, the ratio of the surface area of the solid metal to the volume of the liquid metal in a static solution, the flow rate in a dynamic solution, the surface conditions of the steel, the content of interstitial impurities such as oxygen and nitrogen in the liquid metal/alloy and the compositions of the solid materials [5]. The temperature of the LBE loop affected the corrosion rate very much, because the solubility of the materials in LBE will increase as the temperature increases. There are two types of corrosion: uniform and local [5]. Uniform corrosion is characterized by the uniform damage at the surface of the solid phase by the LBE. For the local corrosion such as intergranular penetration, the liquid metal can penetrate into the solid materials in the areas where zones with a high density of crystal structure defects reaching the surface. The analyze above has neglected the effect of the velocity of the liquid metals, in fact when LBE flows over the structural material surfaces, the corrosion processes will be accelerated, which is termed as flow accelerated corrosion (FAC). The combination of dissolution and the effect of FAC results in four main types of flow accelerated corrosion: mass transport-controlled corrosion, phase transport-controlled corrosion, erosion-corrosion and cavitation-corrosion [6]. Mass transport controlled corrosion occurs when the flow velocity is low. The mass transfer rate (combined effects of convection and diffusion) is less than the dissolution rate of the solid metal into the liquid metal at the interface, and the corrosion rate is determined by the mass transfer rate. For these cases, the corrosion product concentration near the solid/liquid interface equals the saturation or equilibrium concentration. For multi-phase flows or highly turbulent flows, the aggressive particles in the liquid moving along the protective film surface or the high shear stress at the interface can strip the protective films or wear them away, reducing the thickness of the protective film and leading to erosion-corrosion. In the areas where the flow changes its direction sharply, such as sudden expansions or elbows, the liquid or the aggressive particles are thrown against the surface of solid metals, which results in a higher wear rate. Cavitation corrosion occurs when there are cavitation bubbles in the flowing liquid metals and the bubbles collapse on solid metal surfaces. The collapse creates micro-jets of the liquid metal to the solid metal surface, producing high local pressure and destroying the surface. This behavior can lead to brief high stress in the solid metal, causing localized corrosion fatigue damage and environmentally assisted micro-fractures of the solid metal.

Large amount of R\&D work has been done to mitigate the corrosion of the structural materials. There have been two approaches for the anti-corrosion so far. One is the traditional oxygen injection (gas-phase oxygen control technique), the other is solid-phase oxygen control technique. Both of the approaches exploit the fact that lead and bismuth are chemically less active than the major components of steels, such as Fe, $\mathrm{Ni}$, and $\mathrm{Cr}$ as well as $\mathrm{Si}$ [7]. So it is possible to maintain a protective oxide film on the surfaces of the structural materials by carefully controlling the oxygen concentration in LBE. If the concentration is too high, the lead and bismuth will be excessive oxidized which may lead to precipitation contamination and other problems. If the concentration is too low, the protective oxide film cannot be formed, and then the structural materials may be exposed to the LBE directly and corroded severely. So the key to the anti-corrosion is precise controlling of the oxygen concentration.

The traditional technique includes the direct injection of oxygen/hydrogen or hydrogen/water gases mixed with an inert carrier gas (helium or argon) and using oxygen-containing compounds with high oxygen pressure placed over the cover gas cavity of the circuit. During the experiments it is found that the gas-phase oxygen control technique has many disadvantages: (1) inefficient, the ion exchange rate between gas and LBE is very low, leading to a long response time and rapid control difficulties; (2) the pressure and tightness of the corresponding lines need to be paid a lot of attention during the design and adjust processes; (3) the excess oxygen in the export of the oxygen injection line may form oxide residues, blocking the lines or migrating and depositing to the other areas. They are difficult to remove and clear; (4) the reaction of excess oxygen and hydrogen generates steam, which needs to be discharged through special drainage pipeline, the process breaks the sealing of the closed loop. The experimental result shows that in long-term operations, a large amount of slag deposition is formed and accumulated on the surfaces of structural materials using the gas-phase oxygen control technique.

A new approach called the solid-phase oxygen control technique was proposed in the development of LBE program [15]. This technique exploits the solid PbO spheroids instead of gaseous oxygen to provide oxygen for the formation of protective oxide layer. The $\mathrm{PbO}$ spheroids are inserted and retained in the mass exchanger internal chamber. The oxygen is released and transported by the coolant flow through solid $\mathrm{PbO}$ spheroids in contact with flowing LBE in the mass exchanger without extra slag generation. The mass and concentration of dissolved oxygen in the LBE loop can be adjusted by the flow rate controlled by the branch valve and the temperature controlled by the heat exchanger wrapping outside of the mass exchanger. Hence, the efficiency of the system can be improved a lot compared with the gas-phase oxygen control technique and rapid control of the oxygen concentration can be realized. The mass exchanger was installed in the coldest area of the loop, so as to avoid the deposition of oxides in other places of the loop. It is obvious the use of mass exchanger (generators of dissolved oxygen) is the effective technological procedure for slag prevention and it is easier for the precise and rapid control of the concentration of oxygen in the loop. 


\section{The fouling factor}

\subsection{The fouling factor on $L B E$ system}

The oxide layer mitigates the corrosion of structural materials, but it introduces new problems as well. The experimental results indicate that during high-oxygen concentration operation or even normal operation, the inside heat transfer surfaces are often subject to fouling by fluid impurities, oxide formation, or other reactions between the liquid metal and the wall materials. The deposition of an oxide layer or scale on the surface can greatly increase the resistance to heat transfer between the fluid and the wall. This effect can be treated by introducing an additional thermal resistance termed the fouling factor. Its value depends on many factors, such as operating temperature, oxygen concentration, and fluid velocity [8].

The fouling behavior has a great impact on the operation of heat exchanger in the LBE loop. Many fouling models have been developed based on the mass balance equation first proposed by Kern and Seaton. In the equation, the rate of fouling deposition depends on the type of fouling mechanism such as, sedimentation and crystallization while the rate of fouling removal depends on both the adhesive strength of the deposit and the shear stress, as well as the system configuration [9]. The investigation indicates that the fouling factor mainly depends on the effect of velocity, film temperature, and the shear stress. This paper is focused on a type of fouling that has received little attention in the past in spite of its importance to liquid metal heat transfer equipment. This fouling is due to oxidation products that formed on heat transfer surfaces exposed to liquid metal.

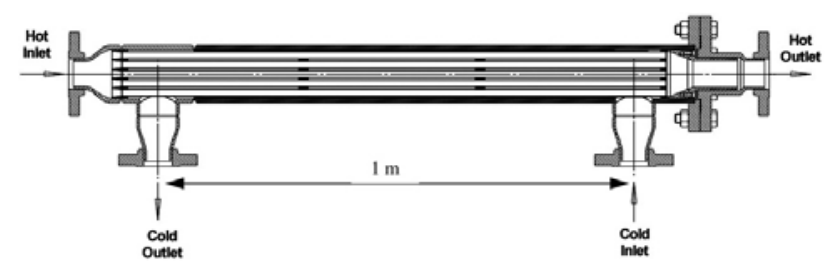

Figure 1. The layout of recuperator.

\subsection{Model derivation of the fouling factor}

The effect of fouling is mainly reflected by the recuperator in the LBE system as shown in Figure 1.

Assuming the heat loss from the recuperator to its surroundings is negligible for the convenience in the derivation processes. Then the heat transferred through the recuperator can be expressed as:

$$
q=m_{h} c_{p, h}\left(T_{h, i}-T_{h, o}\right)=m_{c} c_{p, c}\left(T_{c, o}-T_{c, i}\right)
$$

where $m$ is the mass flow rate, $c_{p}$ means the specific heat, and $T$ stands for the fluid temperature. The distance between the cold inlet and outlet is about 20 times of the inlet/outlet diameter, so the recuperator can be treated as a long, thin heat exchanger, then the cross flow near the inlet and outlet can be neglected. The heat exchanger basically operates in a counter-current configuration. Then the heat transfer rate can be calculated with the equation:

$$
q=U A \Delta T_{L M T D}
$$

where $U$ is the overall heat transfer coefficient, $A$ means the heat transfer area, and $\Delta T_{L M T D}$ stands for the logarithmic mean temperature difference. The overall heat transfer coefficient $k$ can be expressed as [1]:

$$
\begin{aligned}
k=\frac{1}{U A}= & \frac{1}{h_{h} A_{h}}+\frac{R_{f, h}}{A_{h}}+\frac{\ln \left(D_{t, o} / D_{t, i}\right)}{2 \pi k L \cdot n} \\
& +\frac{R_{f, \mathrm{c}}}{A_{c}}+\frac{1}{h_{c} A_{c}}
\end{aligned}
$$

where $R_{f}$ is the fouling factor, $L$ means the length of the tubes, $n$ stands for the number of tubes, $D_{t, o}$ and $D_{t, i}$ are I.D. and O.D. of the tubes, respectively.

Here $A_{n}$ is introduced for convenience and it is defined as:

$$
A_{n}=\frac{A_{h} A_{c}}{A_{h}+A_{c}}
$$

Since both sides of the tubes are contacting the same liquid-metal, we can define the fouling factor on two area bases as:

$$
k=\frac{1}{U A}=\frac{1}{h_{h} A_{h}}+\frac{\ln \left(D_{t, o} / D_{t, i}\right)}{2 \pi k L \cdot n}+\frac{1}{h_{c} A_{c}}+\frac{R_{f}}{A_{n}}
$$

The assumption of fully developed turbulent flow is made in both the tube side and shell side of the recuperator in order to calculate the coefficient of convective heat transfer $h$. The coefficients of convective heat transfer $h_{h}$ and $h_{c}$ can be calculated by the Seban Shimazaki formula [10] and the empirical equation [11] respectively as follows:

$$
\begin{gathered}
N u_{h}=5.0+0.025\left(\operatorname{Re}_{m} P r_{m}\right)^{0.8} \\
N u_{c}=6.66+3.126\left(\frac{P}{D_{t, o}}\right)+1.184\left(\frac{P}{D_{t, o}}\right)^{2} \\
+0.0155\left(\Psi \operatorname{Re}_{m} P r_{m}\right)^{0.86}
\end{gathered}
$$

where $P$ is the pitch between two centers of the tubes and $\Psi$ is the ratio between eddy diffusivities of heat and momentum, the subscript $m$ indicates that the fluid properties are evaluated at the mean bulk temperature. $\Psi=1$ is adopted in the formula above through the comparison of analytical predictions with experimental results carried out by Dwyer [11]. Then from Eqs. (1), (2), and (4), the fouling factors $R_{f}$ can be expressed as:

$$
\begin{aligned}
R_{f}= & \left(\frac{\Delta T_{\text {LMTD }}}{m_{h} c_{p, h}\left(T_{h, i}-T_{h, o}\right)}-\frac{1}{h_{h} A_{h}}-\frac{\ln \left(D_{t, o} / D_{t, i}\right)}{2 \pi k L \cdot n}\right. \\
& \left.-\frac{1}{h_{c} A_{c}}\right) A_{n}
\end{aligned}
$$

The fouling factors $R_{f}$ can be calculated through the Eq. (7) with the experimental data and design parameters, which enables us to investigate the effects of thermal and chemical factors such as temperature, flow rate, and oxygen concentration on the fouling factor and the heat transfer processes between liquid metal and pipes. 


\section{Experimental results}

The gas-phase oxygen control system was adopted in the experiments [8]. The system consists of oxygen sensors, gas injection devices, and filters. The system changes the concentration of oxygen in LBE through injection of oxygen-helium mixture and hydrogen-helium mixture. When oxygen-helium mixture is introduced into the loop the oxygen concentration will increase, while the dissolved oxygen will be removed when hydrogen-helium mixture is injected into the liquid metal. The oxygen sensors are designed and installed in the loop to measure the oxygen concentration in the liquid metal.

The experiments were conducted with three different oxygen concentrations of $4.6 \times 10^{-7} \mathrm{wt} \%, 1.63 \times$ $10^{-5} \mathrm{wt} \%$,and $5.84 \times 10^{-5} \mathrm{wt} \%$, typically representing the low, medium and high oxygen content in LBE within the temperature range $395-520{ }^{\circ} \mathrm{C}$. The results of the experiments are shown in Table $\mathbf{1}$. The results show that the fouling factor increases significantly with the oxygen concentration in the range of $1.0 \times 10^{-5} \mathrm{wt} \%$, because the oxide layers begin to form in this range of oxygen concentration.

The following expression can be got through the experimental data:

$$
R_{f}=2 \times 10^{-4} C_{o}^{0.1514}
$$

where $C_{o}$ is the oxygen concentration in wt\%. Figure 2 shows the experimental data and the correlation with fouling factor.

Table 1. The experimental data under different oxygen content.

\begin{tabular}{ccccccc}
\hline & $T_{\mathrm{c}, i}$ & $T_{\mathrm{c}, o}$ & $T_{\mathrm{h}, i}$ & $T_{\mathrm{h}, o}$ & $V$ & $\begin{array}{l}t \\
\left({ }^{\circ} \mathrm{C}\right)\end{array}$ \\
& $\left({ }^{\circ} \mathrm{C}\right)$ & $\left({ }^{\circ} \mathrm{C}\right)$ & $\left({ }^{\circ} \mathrm{C}\right)$ & $\left(\mathrm{m}^{3} / h\right)$ & $(h)$ \\
\hline 1 & 436.9 & 495.8 & 519.8 & 461.1 & 4.38 & 18.2 \\
2 & 395.1 & 430.0 & 446.3 & 410.5 & 3.87 & 39.3 \\
3 & 403.9 & 417.7 & 426.4 & 412.6 & 4.79 & 24.0 \\
\hline
\end{tabular}

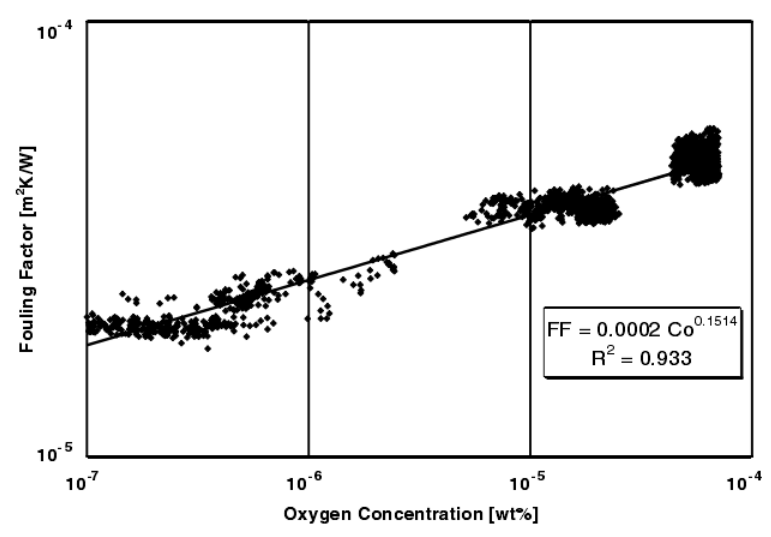

Figure 2. Fouling factor vs. oxygen concentration.

\section{Discussion}

The experimental results show that the fouling factor increases with the increase of oxygen concentration. That is the thermal resistance of the whole heat transfer process increases with the increase of oxygen concentration. Because the oxygen dissolved in LBE can form oxide layers on the pipe wall, which not only increases the thermal resistance for heat conduction, but also makes it more difficult for the coolant to wet pipe surfaces, both of which contribute to the fouling. Following an extensive amount of experimental investigation on the effects of wetting on liquid metal heat transfer, the wetting or lack of wetting does not significantly affect liquid-metal heat transfer. However, non-wetting combinations of liquid metals and solid surfaces can suffer more readily from gas-entrainment problems at the solid-liquid interface; impurities and particles can more easily become trapped at a non-wetting solid-liquid interface, which will greatly reducing heat transfer [12]. The oxide-layer structure of steel in a liquid-lead alloy with oxygen control principally depends on steel composition, temperature, and hydraulic factors. For temperatures below $550^{\circ} \mathrm{C}$, a 10-20 $\mu \mathrm{m}$ external magnetite $\left(\mathrm{Fe}_{3} \mathrm{O}_{4}\right)$ layer and a compact internal spinel $\left((\mathrm{Fe}, \mathrm{Cr})_{\mathrm{x}} \mathrm{O}_{4}\right)$ layer of roughly equal thickness are generated on the pipe surface [13]. This duplex-layer can protect steels from corrosion, but it introduces more thermal resistant as well.

The thermal conductivity of magnetite at temperatures within the range 340-657 $\mathrm{K}$ and the spinel at temperatures from 100 to $1325^{\circ} \mathrm{C}$ can be given by the following equations [14]:

$$
\begin{aligned}
& k_{\text {magnetite }}=4.23-1.37 \times 10^{-3} T(\mathrm{w} / \mathrm{mK}) \\
& k_{\text {spinel }}=50.78 T^{-0.36}(\mathrm{w} / \mathrm{mK})
\end{aligned}
$$

where $T$ is in unit $\mathrm{K}$ in Eq.(9) and in unit ${ }^{\circ} \mathrm{C}$ in Eq.(10) respectively. The experimental Loop had been in operations for a very long time, so the growth of the oxide layers on the pipe walls should have become very small. For the durations and temperatures of the tests, it is reasonable to assume that the oxides do not change, and the approximate total thickness is about $20 \mu \mathrm{m}$. The thermal resistances for conduction, convection and fouling can be calculated from the experimental data and empirical equations.

The experimental results confirm that the wetting condition of the inside surface of the pipes is worsened by the dissolved oxygen. Figure 3 shows the interior of the pipe operated in the low oxygen concentration. The traces of the frozen LBE indicate the pipe has once experienced good wetting under the low oxygen level. Figure 4 shows the interior of the pipe operated in higher oxygen concentration, the wetting condition begin to get worse. Figure 5 shows that after the operation at the highest oxygen concentration, the pipe showing significant oxidation and evidence of non-wetting. It is obvious the extent of adherence decreases significantly with the high oxygen concentration. So the introduction of oxygen does reduce wetting in the pipe over time.

The fouling effect will contribute more to the total thermal resistance if the concentration of the dissolved oxygen gets higher and it is not difficult to predict that the effect will reach its maximum as the oxygen in LBE reach the solubility limit. 


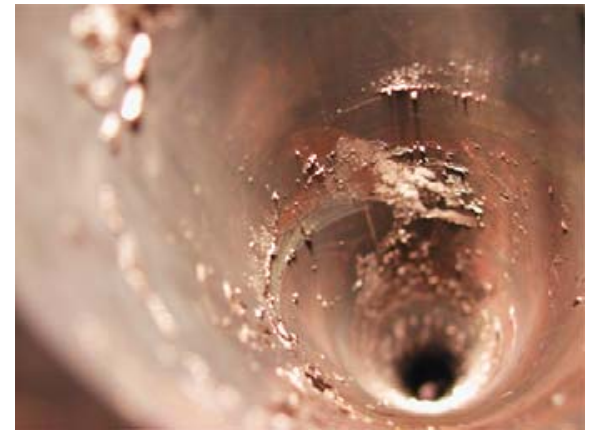

Figure 3. The inside view of the pipe after the operation at low oxygen concentration.

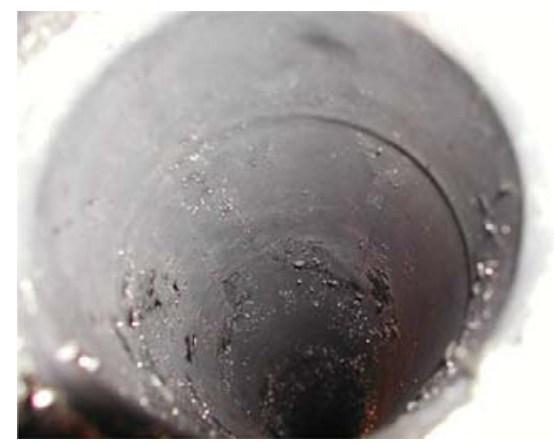

Figure 4. The inside view of the pipe after the operation at middle oxygen concentration.

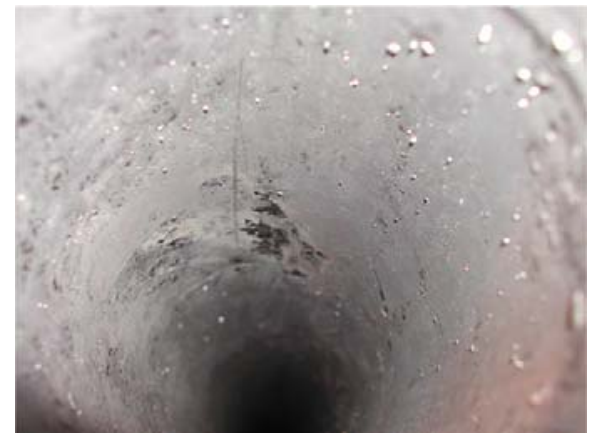

Figure 5. The inside view of the pipe after the operation at high oxygen concentration.

\section{Conclusions}

The active oxygen control technique can mitigate the corrosion processes of structure materials effectively, but the traditional gas-phase oxygen control technique has a lot of side effect to the system. The fouling effect cannot be avoided in the application of liquid metal in fast reactor and spent fuel transmutation systems. The effect increases with the oxygen concentration increase in LBE. The introduction of oxygen will enhance the generation of oxide film and therefore reduce the amount of wetting in the pipe over time. The non-wetting condition and impurities and gas trapped at non-wetting solid-liquid interfaces are the primary factors in reducing heat transfer and increasing fouling. The fouling will reach maximum as the liquid-metal is saturated with oxygen. The correlation between the fouling factor and oxygen concentration can be used to evaluate the fouling caused by oxygen in the liquid-metal. The application of LBE as a coolant in nuclear system requires a comprehensive consideration of the effects of oxygen on corrosion resistance and Thermal Hydraulic effects.

\section{Acknowledgments}

This work was supported by the National Natural Science Foundation of China (91326108), and the Strategic Priority Research Program of the Chinese Academy of Sciences (XDA03040000).

\section{References}

[1] F. P. Incropera and D. P. Dewitt, Fundamentals of Heat and Mass Transfer, John Wiley \& Sons, New York, (1996), p.585.

[2] J. Zhang, N. Li, Y. Chen and A. E. Rusanov, Corrosion behaviors of US steels in flowing lead-bismuth eutectic (LBE), Journal of Nuclear Materials 336 (2005), pp. 1-10.

[3] J. Zhang and N. Li, Corrosion/precipitation in non-isothermal and multi-modular LBE loop systems. Journal of Nuclear Materials 326 (2004), pp. 201-210.

[4] J. R. Weeks, Mechanisms of Liquid Metal Corrosion, Fourth NASA-AEC Liquid Metal Corrosion Meeting, CONF-428, October 2, 1963.

[5] E. M. Lyutyi, Sov. Union Mater. Sci. 24 (1988), p. 441.

[6] E. Heitz, Corrosion 47 (2) (1991), p.135.

[7] X. He, N. Li and M. Mineev, A kinetic model for corrosion and precipitation in non-isothermal LBE flow loop. Journal of Nuclear Materials 297 (2001), pp. 214-219.

[8] F. Niu, Robert Candalino and N. Li, Effect of oxygen on fouling behavior in lead-bismuth coolant systems, Journal of Nuclear Materials 366 (2007), pp. 216-222.

[9] D. Q. Kern and R. E. Seaton, Br. Chem. Eng. 4 (5) (1959), pp. 258-262.

[10] R. A. Seban and T. T. Shimazaki, Trans. ASME 73 (1951), p.803.

[11] O. J. Foust, Sodium-NaK, Engineering Handbook II, (1976), pp. 105-106, 204.

[12] S. Kakac, R. K. Shah and W. Aung, Handbook of Single-phase Convective Heat Transfer, (1987), pp. 8.1, 8.9, 8.23.

[13] F. Balbaud-Celerier, A. Terlain et al., Report Technique RT-SCCME 630, CEA Report, (2003).

[14] J. MUlgaard and W. W. Smeltzer, J. Appl. Phys. 42 (9) (1971), p.3644.

[15] P. N. Martynov, R. S. Askhadullin et al., Lead-bismuth and lead coolants in new technologies of reprocessing of liquids and gases, Proceedings of the Fast Neutrons Reactors Conference, Obninsk, Russian Federation, (2003). 